\title{
Effects of accelerated storage on the quality of kenaf seed oil in chitosan-coated high methoxyl pectin-alginate microcapsules
}

\begin{abstract}
The objective of this research was to study the oxidative stability and antioxidant properties of microencapsulated kenaf (Hibiscus cannabinus L.) seed oil (MKSO) produced by coextrusion technology upon accelerated storage. The combination of sodium alginate, high methoxyl pectin, and chitosan were used as shell materials. The oxidative stability of the kenaf seed oil was determined by iodine value, peroxide value, $\mathrm{p}$-Anisidine value, total oxidation (TOTOX), thiobarbituric acid reactive substances assay, and free fatty acid content. Total phenolic content, 2,2'-azino-bis(3-ethylbenzothiazoline-6-sulphonic acid) cation radical-scavenging assay and 2,2-diphenyl-1-picrylhydrazyl radical scavenging assay were used to examine the antioxidant properties of oils. Oxidative stability tests showed that bulk kenaf seed oil (BKSO) was oxidized significantly higher $(\mathrm{P}<0.05)$ than MKSO. The total increment of TOTOX value of BKSO was $165.93 \%$ significantly higher $(\mathrm{P}<0.05)$ than MKSO. Co-extrusion technology has shown to be able to protect kenaf seed oil against lipid oxidation and delay the degradation of natural antioxidants that present in oil during storage.
\end{abstract}

Keyword: Antioxidant activity; Co-extrusion technology; Correlation; Encapsulator B-390; Oxidative stability 